\title{
Predictors of Recurrent In-Stent Restenosis After Paclitaxel-Coated Balloon Angioplasty
}

\author{
Hiroshi Koiwaya, MD, PhD; Nozomi Watanabe, MD, PhD; Nehiro Kuriyama, MD, PhD; \\ Shun Nishino, MD; Kenji Ogata, MD; Toshiyuki Kimura, MD; Tatsuya Nakama, MD; \\ Hirohide Matsuura, MD, PhD; Makoto Furugen, MD, PhD; Yoshisato Shibata, MD
}

\begin{abstract}
Background: Although paclitaxel-coated balloon (PCB) angioplasty is an effective procedure for in-stent restenosis (ISR) after coronary stenting, recurrent ISR after PCB angioplasty still occurs. The aim of this study was to evaluate the predictors of recurrent ISR after PCB angioplasty for ISR.
\end{abstract}

\begin{abstract}
Methods and Results: A total of 157 ISR lesions treated with PCB angioplasty from January 2014 to May 2015 were retrospectively examined. Recurrent ISR was judged on 6-month follow-up angiography. Clinical, angiographic and procedural parameters were evaluated as possible predictors of recurrent ISR. Recurrent ISR occurred in $13.9 \%$ of lesions after PCB angioplasty. On multivariate analysis the following independent predictors of recurrent ISR were identified: (1) smaller acute gain after initial ballooning (OR, 3.06; 95\% Cl: 1.08-8.71; $\mathrm{P}=0.04)$; (2) geographic mismatch between PCB position and initial ballooning (OR, 5.59; 95\% Cl: 1.64-19.1; $\mathrm{P}=0.006$ ); and (3) use of percutaneous transluminal coronary rotational atherectomy (PTCRA) at primary percutaneous coronary intervention (PCl; OR, 5.53; 95\% Cl: 1.89-16.2; $\mathrm{P}=0.002$ ).
\end{abstract}

Conclusions: Optimal expansion at initial ballooning before PCB angioplasty and careful positioning of PCB are important technical tips to prevent recurrent ISR after PCB angioplasty. Recurrent ISR occurred more frequently in severely calcified lesions that required PTCRA at primary PCl.

Key Words: In-stent restenosis; Paclitaxel-coated balloon angioplasty; Percutaneous coronary intervention; Recurrent in-stent restenosis

$\mathbf{N}$ ew-generation drug-eluting stents (DES) have gained widespread use due to their extraordinarily low rates of coronary restenosis and stent thrombosis, and lower risk of death. ${ }^{1}$ The increased use of DES, however, has led to the expansion of percutaneous coronary intervention (PCI) indication to higher risk patients with or without complex lesions, which subsequently causes an increase in risk of in-stent restenosis (ISR). ${ }^{2}$ Furthermore, the optimal PCI strategy for ISR is still controversial.,3 In the treatment for ISR, conventional balloon angioplasty (BA) may induce recurrent ISR with high probability. ${ }^{4}$ As a result, repeat DES implantation for ISR, the so-called "stent-in-stent" procedure, has been widely performed. Repeat DES deployment, however, causes a new issue of multiple stent layers in the coronary arteries. ${ }^{5}$ BA using a balloon coated with anti-proliferative drugs, based on the concept of intramural drug delivery without polymer or metal, is a promising therapy for ISR, because the main cause of ISR is neointimal proliferation. ${ }^{6}$ One of the benefits of drug-coated balloon (DCB) angioplasty is the metal-free procedure, which enables avoidance of addi- tional stent implantation and which facilitates repeat DCB angioplasty.,5-10 Several randomized trials have reported that paclitaxel-coated balloon (PCB) angioplasty for bare metal stent (BMS)-ISR or DES-ISR provides better clinical and angiographic results compared with conventional $\mathrm{BA}$, such as significantly lower recurrent restenosis and target lesion revascularization (TLR). ${ }^{711-13}$ Hence, PCB angioplasty is expected to be a promising therapy for ISR. Two meta-analyses of the effect of DCB to treat ISR showed that DCB reduces the risk of major adverse cardiac events, compared with not only conventional BA but also additional DES implantation,, $\mathbf{8 9}$ and 1 meta-analysis of PCI strategies for ISR also demonstrated the better angiographic and clinical outcomes without the addition of metal stent layers. ${ }^{10}$ Studies on the long-term clinical outcomes and safety after PCB angioplasty for both BMSISR and DES-ISR, compared with conventional BA, reported no stent thrombosis. ${ }^{13,14}$ Although PCB angioplasty was expected to be an alternative to repeat DES implantation for ISR, recurrent ISR still occurs in the clinical setting. In the present study, we evaluated the inde-

Received January 29, 2017; revised manuscript received March 13, 2017; accepted March 15, 2017; released online April 28, 2017 Time for primary review: 23 days

Department of Cardiology, Miyazaki Medical Association Hospital Cardiovascular Center, Miyazaki, Japan

Mailing address: Hiroshi Koiwaya, MD, PhD, Department of Cardiology, Miyazaki Medical Association Hospital Cardiovascular Center, 738-1 Funado, Shinbeppu-chou, Miyazaki 880-0834, Japan. E-mail: koiwaya.hiroshi@gmail.com

ISSN-1346-9843 All rights are reserved to the Japanese Circulation Society. For permissions, please e-mail: cj@j-circ.or.jp 
pendent predictors of recurrent ISR after PCB angioplasty.

\section{Methods}

\section{Patients and Study Design}

This study was a single-center retrospective analysis of a prospectively maintained database at Miyazaki Medical Association Hospital Cardiovascular Center. From January 2014 to May 2015, 130 patients with ISR after stent implantation were enrolled in this study (Figure 1). All patients presented with stable angina. PCB angioplasty was performed for 157 lesions and follow-up coronary angiography at 6 months for 151 lesions (follow-up rate, $96.2 \%$ ). Patients were then divided into 2 groups according to presence of recurrent ISR: no recurrent ISR $(n=130$ lesions) and recurrent ISR ( $\mathrm{n}=21$ lesions). The study protocol was developed in accordance with the Declaration of Helsinki, and was approved by the hospital's ethics committee. All patients gave informed consent before the procedure.

\section{PCI}

The PCI were performed with a 6-Fr or 7-Fr guiding catheter via the radial or femoral artery. Standard interventional techniques were used and performed according to practice guidelines. During PCI, heparin was used to maintain activated clotting time $>250 \mathrm{~s}$. All patients were prescribed dual antiplatelet therapy, that is, aspirin $(100 \mathrm{mg}$ daily) and either ticlopidine ( $200 \mathrm{mg}$ daily) or clopidogrel ( $75 \mathrm{mg}$ daily), for $\geq 3$ months in accordance with the instructions for use. Baseline coronary angiography after intracoronary nitroglycerin was recorded in multiple views. Intravascular ultrasound (IVUS) or optical coherence tomography (OCT) was used to determine the diameter and length of the initial BA or PCB angioplasty. According to the instructions for use, initial BA with a non-compliant balloon, scoring balloon, or both was performed prior to inflating the PCB (SeQuent Please balloon catheter, B. Braun Melsungen, Vascular Systems, Berlin, Germany). As a scoring balloon, Lacrosse ${ }^{\circledR}$ NSE balloon catheter (Goodman, Nagoya, Japan), AngioSculpt ${ }^{\circledR}$ PTCA balloon catheter (AngioScore, Fremont, CA, USA), or Cutting balloon catheter (Boston Scientific, Marlborough, MA, USA) was used. The length of the PCB was chosen to exceed the lesion dilated by the initial balloon by $\geq 2 \mathrm{~mm}$ at the proximal and distal margins. The PCB was inflated for $\geq 30$ s as per the instructions for use.

\section{Angiography}

Coronary angiography was performed before and after the successful procedure, and at 6-month follow-up. Quantitative coronary angiography (QCA) was performed with the CAAS 5.9 (Pie Medical Imaging BV, Maastricht, the Netherlands). Coronary angiography after intracoronary nitroglycerin was recorded in multiple views. Reference diameter (RD), minimum lumen diameter (MLD), percentage diameter stenosis (\%DS), and lesion length were measured before and after the procedure and at 6-month follow-up. Acute gain-1 and acute gain-2 were defined as MLD immediately after initial BA minus that at baseline, and as MLD immediately after PCB angioplasty minus that after initial BA, respectively. The expansion rate at initial ballooning was defined as MLD immediately after initial BA divided by RD at baseline. Late lumen loss (LLL) was defined as the difference between the MLD
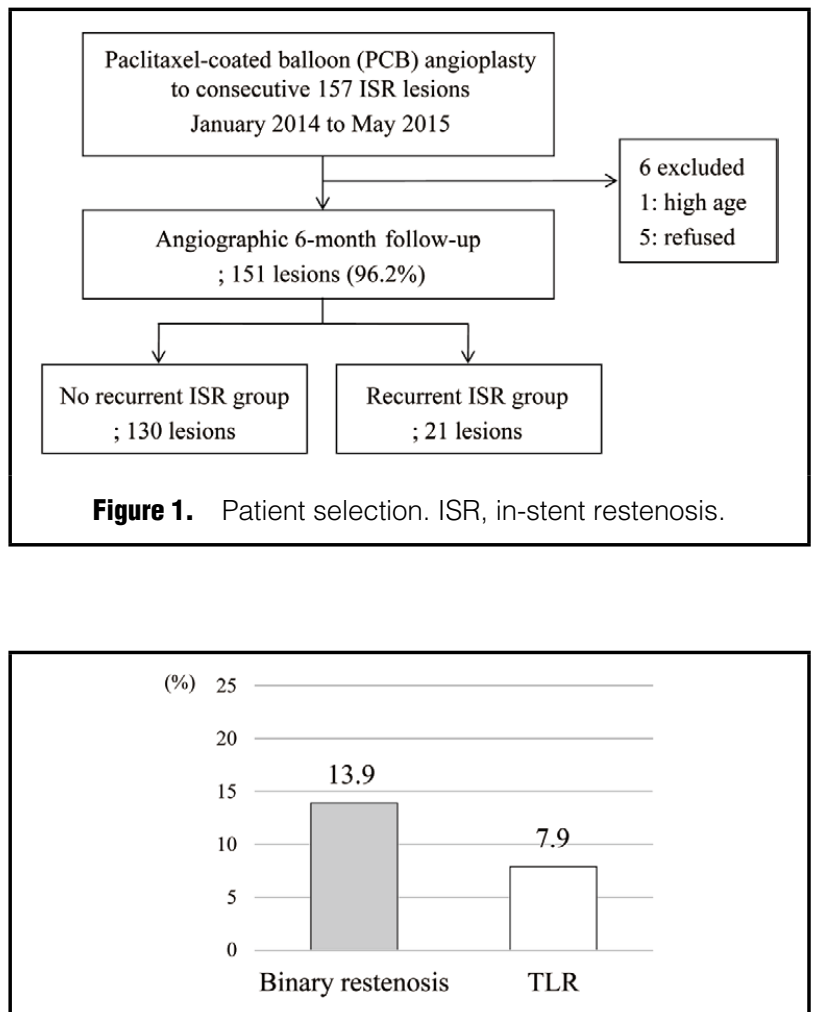

Figure 2. Angiography at 6-month follow-up. TLR, target vessel revascularization.

immediately after PCB angioplasty and that at 6-month follow-up. These measurements were performed from shoulder to shoulder (in-stent) where the PCB was dilated.

\section{Follow-up Protocol and Definitions}

Clinical and angiographic follow-up were performed at 6 months after successful PCB angioplasty. Follow-up coronary angiography was performed earlier than 6 months in the case of clinically indicated ischemia. Binary restenosis at follow-up was defined as stenosis occupying $>50 \%$ of the diameter. TLR was defined as any recurrent PCI or coronary bypass surgery due to restenosis ( $\% \mathrm{DS} \geq 50 \%$ ) associated with symptoms or objective signs of ischemia. ${ }^{2}$

\section{Statistical Analysis}

Statistical analysis was performed on an intension-to-treat basis. Continuous variables are expressed as mean \pm SD. Categorical values are presented as numbers with relative percentages or SD. Parametric Student's t-test was used to compare continuous variables, and chi-squared test for categorical values. Multivariate logistic regression analysis was performed to evaluate the threshold of expansion rate after initial BA to detect whether it could be related to the recurrent ISR, and to identify independent predictors of recurrent ISR after PCB angioplasty. Variables were selected according to significance on univariate analysis (defined as $\mathrm{P}<0.05$ ), but some factors were not included because of strong correlation with other parameters. Cumulative 6-month freedom from ISR was assessed using the Kaplan-Meier method. All statistical analysis was performed using SPSS version 19 (IBM Corporation, Somers, NY, USA). 


\begin{tabular}{|c|c|c|c|}
\hline & $\begin{array}{l}\text { No recurrent ISR } \\
\text { ( } n=130 \text { lesions) }\end{array}$ & $\begin{array}{l}\text { Recurrent ISR } \\
\text { ( } n=21 \text { lesions) }\end{array}$ & $P$ value \\
\hline Age (years) & $68.6 \pm 8.8$ & $67.9 \pm 5.0$ & 0.58 \\
\hline Male & $111(85.4)$ & $15(71.4)$ & 0.10 \\
\hline \multicolumn{4}{|l|}{ Coronary risk factor } \\
\hline Hypertension & $108(83.1)$ & $20(95.2)$ & 0.13 \\
\hline Diabetes mellitus & $66(50.8)$ & $10(47.6)$ & 0.79 \\
\hline Dyslipidemia & $100(76.9)$ & $14(66.7)$ & 0.31 \\
\hline Current smoker & $35(26.9)$ & 7 (33.3) & 0.54 \\
\hline Previous MI & 77 (59.2) & $8(38.1)$ & 0.07 \\
\hline PAD & $5(3.8)$ & $3(14.3)$ & 0.08 \\
\hline CKD, HD & $3(2.3)$ & $1(4.8)$ & 0.45 \\
\hline \multicolumn{4}{|l|}{ Target lesion } \\
\hline LMT & $2(1.5)$ & $1(4.8)$ & 0.36 \\
\hline LAD & $71(54.6)$ & $8(38.1)$ & 0.16 \\
\hline LCX & $22(16.9)$ & $2(9.5)$ & 0.31 \\
\hline $\mathrm{RCA}$ & $35(26.9)$ & $10(47.6)$ & 0.05 \\
\hline \multicolumn{4}{|l|}{ Restenotic stent type } \\
\hline DES & $113(86.9)$ & $19(90.5)$ & 0.49 \\
\hline BMS & $17(13.1)$ & $2(9.5)$ & 0.49 \\
\hline \multicolumn{4}{|l|}{ ISR } \\
\hline Focal & 94 (72.3) & $11(52.4)$ & 0.07 \\
\hline Diffuse & $36(27.7)$ & $9(42.9)$ & 0.16 \\
\hline ISR-CTO & $6(4.6)$ & $1(4.8)$ & 0.66 \\
\hline Time to ISR (months) & $48.5 \pm 40.6$ & $44.1 \pm 39.7$ & 0.65 \\
\hline Previous stent diameter $(\mathrm{mm})$ & $2.93 \pm 0.42$ & $2.86 \pm 0.43$ & 0.46 \\
\hline Previous stent diameter $\leq 2.5 \mathrm{~mm}$ & $39(30.0)$ & 7 (33.3) & 0.76 \\
\hline No. repeated stents $\geq 2$ & $39(30.0)$ & $4(19.0)$ & 0.30 \\
\hline Primary $\mathrm{PCl}$ for ACS & $43(33.1)$ & $3(14.3)$ & 0.08 \\
\hline PTCRA at primary PCl & $20(15.4)$ & $10(47.6)$ & 0.002 \\
\hline
\end{tabular}

Data given as mean \pm SD or $\mathrm{n}(\%)$. ACS, acute coronary syndrome; BMS, bare metal stent; CKD, chronic kidney disease; CTO, chronic total occlusion; DES, drug-eluting stent; HD, hemodialysis; ISR, in-stent restenosis; LAD, left anterior descending artery; LCX, left circumflex; LMT, left main trunk; MI, myocardial infarction; PAD, peripheral arterial disease; $\mathrm{PCl}$, percutaneous coronary intervention; PTCRA, percutaneous transluminal coronary rotational atherectomy; RCA, right coronary artery.

\section{Results}

\section{Recurrent Restenosis After PCB Angioplasty}

Angiography After PCB Angioplasty The rates of binary restenosis and TLR after PCB angioplasty were $13.9 \%$ and $7.9 \%$ of lesions, respectively (Figure 2).

Baseline Characteristics A total of 151 lesions (96.2\%) after PCB angioplasty were analyzed in this study. The patients consisted of 109 men and 21 women with a mean age of $68.5 \pm 8.4$ years. A total of 130 lesions $(86.1 \%)$ were enrolled in the no recurrent ISR group and 21 lesions $(13.9 \%)$ in the recurrent ISR group (Figure 1). There were no significant differences in baseline patient characteristics between the 2 groups (Table 1). With regard to baseline restenotic and angiographic characteristics (Table 1), no significant differences were observed between the 2 groups, including in DES-ISR ( $86.9 \%$ vs. $90.5 \%, \mathrm{P}=0.49$ ), focal ISR ( $72.3 \%$ vs. $52.4 \%, \mathrm{P}=0.07)$, previous final stent diameter $<2.5 \mathrm{~mm}(30.0 \%$ vs. $33.3 \%, \mathrm{P}=0.76)$, number of repeated stents $>2(30.0 \%$ vs. $19.0 \%, \mathrm{P}=0.30)$, and primary $\mathrm{PCI}$ for acute coronary syndrome ( $33.1 \%$ vs. $14.3 \%, \mathrm{P}=0.08)$. Time to ISR ( $48.5 \pm 40.6$ months vs. $44.1 \pm 39.7$ months, $\mathrm{P}=0.65$ ) and the previous stent diameter $(2.93 \pm 0.42 \mathrm{~mm}$ vs. $2.86 \pm 0.43 \mathrm{~mm}, \mathrm{P}=0.46$ ) were also similar between the 2 groups. Rate of percutaneous transluminal coronary rotational atherectomy (PTCRA) at primary PCI (15.4\% vs. $47.6 \%, \mathrm{P}=0.002$ ) was significantly higher in the recurrent ISR group than in the no recurrent ISR group. The rate of recurrent ISR was $47.6 \%$ when PTCRA was used at primary PCI.

Changes After PCB Angioplasty Baseline QCA data were similar between the 2 groups (Table 2). Lesion length trended longer in the recurrent ISR group $(19.0 \pm 6.28 \mathrm{~mm}$ vs. $21.8 \pm 8.96 \mathrm{~mm}, \mathrm{P}=0.08)$, but did not reach statistical significance. After PCB angioplasty, \%DS was significantly higher in the recurrent ISR group than in the no recurrent ISR group $(10.9 \pm 11.1 \%$ vs. $18.6 \pm 13.8 \%, \mathrm{P}=0.005)$, and MLD was significantly lower in the recurrent ISR group than in the other group $(2.51 \pm 0.45 \mathrm{~mm}$ vs. $2.12 \pm 0.44 \mathrm{~mm}$, $\mathrm{P}<0.001)$. Acute gain-1 after initial BA $(1.73 \pm 0.44 \mathrm{~mm}$ vs. $1.40 \pm 0.52 \mathrm{~mm}, \mathrm{P}=0.002)$ and expansion rate after initial ballooning $>80 \%(63.1 \%$ vs. $33.3 \%, \mathrm{P}=0.01)$ were significantly lower in the recurrent ISR group. Acute gain-2 after PCB angioplasty $(0.26 \pm 0.29 \mathrm{~mm}$ vs. $0.30 \pm 0.44 \mathrm{~mm}, \mathrm{P}=0.63)$ and type of initial balloon catheter before $\mathrm{PCB}$ angioplasty (non-compliant balloon catheter: $32.3 \%$ vs. $38.1 \%, \mathrm{P}=0.60$ ) were similar between the 2 groups. Geographic mismatch was observed significantly more frequently in the recurrent 
Table 2. Change in QCA and Procedural Characteristics After PCB Angioplasty

\begin{tabular}{|c|c|c|c|}
\hline & $\begin{array}{l}\text { No recurrent ISR } \\
\text { ( } n=130 \text { lesions) }\end{array}$ & $\begin{array}{l}\text { Recurrent ISR } \\
\text { ( } n=21 \text { lesions) }\end{array}$ & $P$ value \\
\hline \multicolumn{4}{|l|}{ Before procedure } \\
\hline$\% D S(\%)$ & $81.1 \pm 11.3$ & $83.6 \pm 10.1$ & 0.34 \\
\hline MLD (mm) & $0.52 \pm 0.32$ & $0.43 \pm 0.27$ & 0.20 \\
\hline $\mathrm{RD}(\mathrm{mm})$ & $2.73 \pm 0.40$ & $2.60 \pm 0.37$ & 0.17 \\
\hline Lesion length (mm) & $19.0 \pm 6.28$ & $21.8 \pm 8.96$ & 0.08 \\
\hline \multicolumn{4}{|l|}{ After initial ballooning } \\
\hline$\% D S(\%)$ & $17.2 \pm 12.3$ & $25.3 \pm 15.4$ & 0.008 \\
\hline MLD (mm) & $2.25 \pm 0.42$ & $1.82 \pm 0.43$ & $<0.001$ \\
\hline Acute gain-1 (mm) & $1.73 \pm 0.44$ & $1.40 \pm 0.52$ & 0.002 \\
\hline \multicolumn{4}{|l|}{ After procedure } \\
\hline$\% D S(\%)$ & $10.9 \pm 11.1$ & $18.6 \pm 13.8$ & 0.005 \\
\hline MLD (mm) & $2.51 \pm 0.45$ & $2.12 \pm 0.44$ & $<0.001$ \\
\hline Acute gain-2 (mm) & $0.26 \pm 0.29$ & $0.30 \pm 0.44$ & 0.72 \\
\hline Expansion rate after initial ballooning (\%) & $82.8 \pm 12.3$ & $74.7 \pm 15.4$ & 0.008 \\
\hline Expansion rate after initial ballooning $\geq 80 \%$ & $82(63.1)$ & 7 (33.3) & 0.01 \\
\hline Type of initial balloon catheter: non-compliant & $42(32.3)$ & $8(38.1)$ & 0.60 \\
\hline Geographic mismatch & $11(8.5)$ & 7 (33.3) & 0.004 \\
\hline \multicolumn{4}{|l|}{ Procedural characteristics } \\
\hline \multicolumn{4}{|l|}{ Initial ballooning } \\
\hline Balloon diameter (mm) & $2.88 \pm 0.42$ & $2.84 \pm 0.47$ & 0.75 \\
\hline Balloon length (mm) & $11.8 \pm 1.87$ & $12.0 \pm 1.75$ & 0.56 \\
\hline Inflation pressure (atm) & $15.9 \pm 4.33$ & $17.5 \pm 3.47$ & 0.11 \\
\hline \multicolumn{4}{|l|}{ PCB } \\
\hline Balloon diameter (mm) & $2.91 \pm 0.38$ & $3.00 \pm 0.50$ & 0.37 \\
\hline Balloon length (mm) & $20.1 \pm 5.39$ & $23.1 \pm 5.93$ & 0.02 \\
\hline Inflation pressure (atm) & $11.8 \pm 2.45$ & $11.4 \pm 2.78$ & 0.47 \\
\hline \multicolumn{4}{|l|}{ 6-month follow-up } \\
\hline$\% D S(\%)$ & $21.0 \pm 15.8$ & $73.0 \pm 19.5$ & $<0.001$ \\
\hline MLD (mm) & $2.24 \pm 0.56$ & $0.70 \pm 0.49$ & $<0.001$ \\
\hline $\mathrm{RD}(\mathrm{mm})$ & $2.80 \pm 0.43$ & $2.63 \pm 0.52$ & 0.11 \\
\hline Late lumen loss (mm) & $0.28 \pm 0.59$ & $1.43 \pm 0.70$ & $<0.001$ \\
\hline
\end{tabular}

Data given as mean \pm SD or $n$ (\%). \%DS, percent diameter stenosis; ISR, in-stent restenosis; MLD, minimum lumen diameter; PCB, paclitaxelcoated balloon; QCA, quantitative coronary angiography; RD, reference diameter.

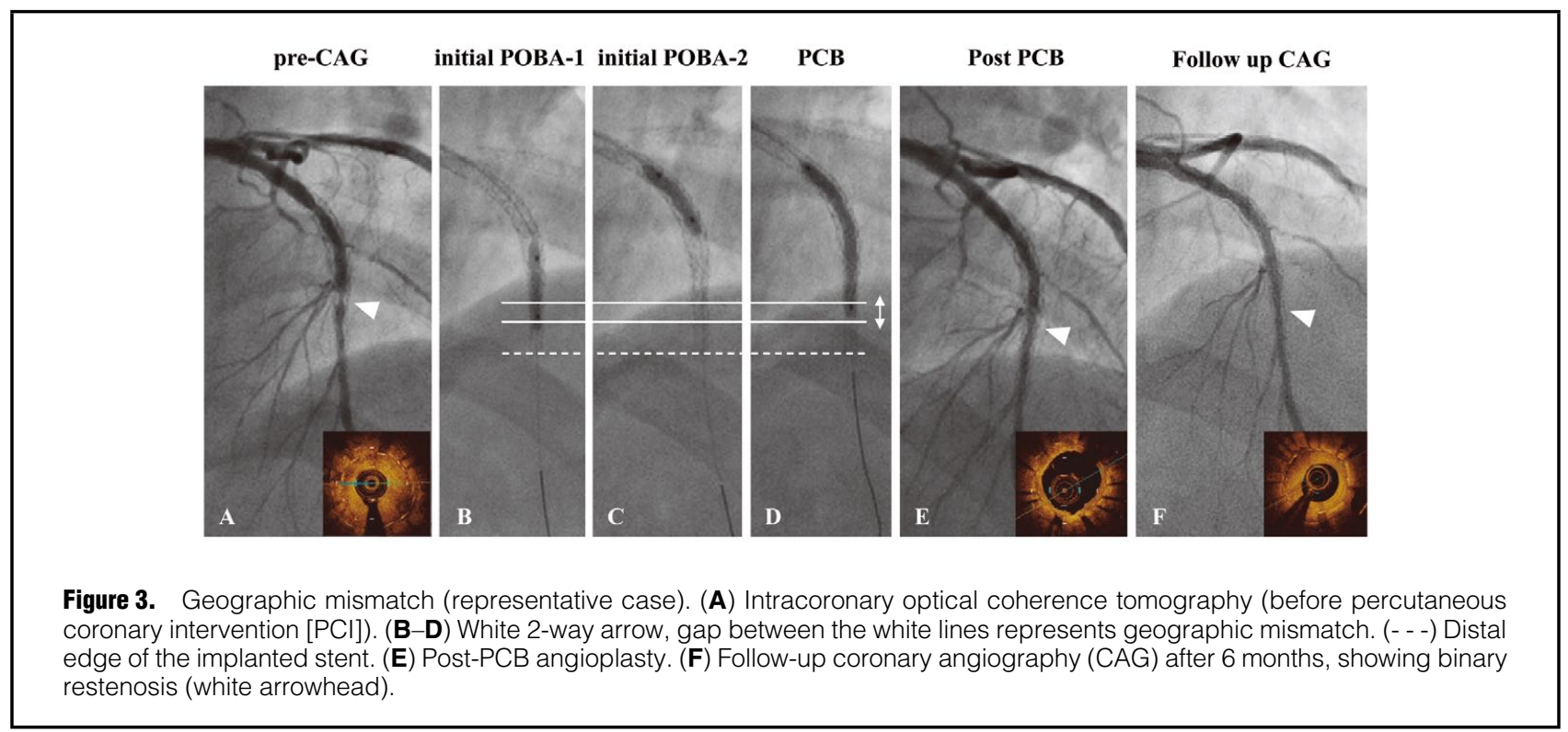




\begin{tabular}{|lcc|}
\hline $\begin{array}{l}\text { Table 3. Independent Predictors of Recurrent ISR After PCB } \\
\text { Angioplasty }\end{array}$ & OR (95\% Cl) & P value \\
\multicolumn{1}{|c|}{ Variable } & OR & 0.04 \\
$\begin{array}{l}\text { Expansion rate at } \\
\text { initial ballooning <80\% }\end{array}$ & $1.08-8.71)$ & 0.04 \\
Geographic mismatch & $5.59(1.64-19.1)$ & 0.006 \\
PTCRA at initial PCl & $5.53(1.89-16.2)$ & 0.002 \\
\hline
\end{tabular}

Abbreviations as in Tables 1,2.

\begin{tabular}{|ccc|}
\hline $\begin{array}{l}\text { Table 4. Initial Expansion Rate and Prognostic Impact on } \\
\text { Recurrent ISR }\end{array}$ & & \\
Variable & OR $\mathbf{( 9 5 \% ~ C l )}{ }^{\dagger}$ & P value $^{\dagger}$ \\
Expansion rate: $80-90 \%$ & $3.83(1.19-12.4)$ & 0.03 \\
Expansion rate: $\geq 90 \%$ & $5.16(1.11-24.1)$ & 0.04 \\
\hline
\end{tabular}

${ }^{\dagger}$ vs. expansion rate $<80 \%$. ISR, in-stent restenosis.

ISR group than in the no recurrent ISR group ( $8.5 \%$ vs. $33.3 \%, \mathrm{P}=0.004)$. A representative case of geographic mismatch at PCB angioplasty followed by recurrent ISR is shown in Figure 3. Geographic mismatch was defined as a difference in the location treated by PCB angioplasty and the location treated by initial BA (Figure 3B,D). In this case, follow-up angiography after 6 months showed binary restenosis (Figure 3F). While balloon diameter and inflation pressure at initial BA and PCB angioplasty were not significantly different between the 2 groups, length of PCB was significantly longer in the recurrent ISR group $(20.1 \pm 5.4 \mathrm{~mm}$ vs. $23.1 \pm 5.9 \mathrm{~mm}, \mathrm{P}=0.02)$. Six-month followup angiography was performed in 151 lesions (follow-up rate, $96.2 \%): \% \mathrm{DS}(21.0 \pm 15.8 \%$ vs. $73.0 \pm 19.5 \%, \mathrm{P}<0.001)$ and late lumen loss $(0.28 \pm 0.59 \mathrm{~mm}$ vs. $1.43 \pm 0.70 \mathrm{~mm}, \mathrm{P}<0.001)$ were significantly higher in the recurrent ISR group, and MLD $(2.24 \pm 0.56 \mathrm{~mm}$ vs. $0.70 \pm 0.49 \mathrm{~mm}, \mathrm{P}<0.001)$ was significantly lower in the recurrent ISR group.

\section{Predictors of Recurrent ISR After PCB Angioplasty}

On multivariate logistic regression analysis to assess the independent predictors of recurrent ISR after PCB angioplasty (Table 3), (1) smaller expansion $(<80 \%)$ at initial ballooning before PCB angioplasty, (2) geographic mismatch, and (3) PTCRA use at primary PCI were independently associated with recurrent ISR after PCB angioplasty (OR, 3.06; 95\%CI: 1.08-8.71, P=0.04; OR, 5.59; 95\% CI: 1.64-19.1, $\mathrm{P}=0.006$; OR, 5.53; 95\% CI: $1.89-16.2, \mathrm{P}=0.002$, respectively).

\section{Prognostic Impact of Initial Balloon Expansion Rate on Recurrent ISR}

To evaluate the effect of initial expansion rate and its prognostic impact on recurrent ISR on multivariate logistic regression analysis, ISR lesions were divided into 3 groups according to expansion rate after initial ballooning on QCA $(<80 \%, 80-90 \%$, and $>90 \%)$. Consequently, freedom from recurrent ISR for expansion rates $80-90 \%$ and $>90 \%$ was significantly higher than that for $<80 \%$ (OR, 3.83; $95 \%$ CI: 1.19-12.4, $\mathrm{P}=0.03$; OR, 5.16; 95\% CI: 1.11-24.1, $\mathrm{P}=0.04$, respectively; Table 4). We also compared the prognosis after PCB angioplasty according to the expansion rate after initial BA. At 6 months after PCB angioplasty, freedom from recurrent ISR was significantly higher for

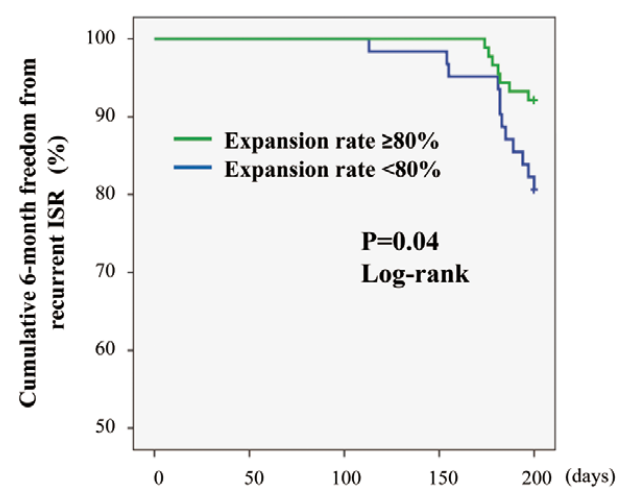

Figure 4. Cumulative 6-month freedom from recurrent instent restenosis (ISR) vs. expansion rate.

expansion rate $\geq 80 \%$ than $<80 \%(92.1 \%$ vs. $80.6 \%, \mathrm{P}=0.04$; Figure 4).

\section{Original Lesion Calcification and PCB}

We compared QCA data between PTCRA use $(n=30)$ and no PTCRA use $(n=121)$ at primary PCI to evaluate the influence of target lesion calcification on recurrent ISR (Table S1). The results of PCB angioplasty were similar between the 2 groups, including expansion rate after initial ballooning $(80.7 \pm 11.5 \%$ vs. $82.0 \pm 13.4 \%, \mathrm{P}=0.63)$, acute gain-1 $(1.76 \pm 0.49 \mathrm{~mm}$ vs. $1.67 \pm 0.45 \mathrm{~mm}, \mathrm{P}=0.36)$, acute gain-2 $(0.22 \pm 0.34 \mathrm{~mm}$ vs. $0.28 \pm 0.31, \mathrm{P}=0.33)$, and MLD after PCB angioplasty $(2.46 \pm 0.53 \mathrm{~mm}$ vs. $2.46 \pm 0.45 \mathrm{~mm}$, $\mathrm{P}=0.99$ ).

\section{Discussion}

In this single-center retrospective study, the following independent predictors of recurrent ISR after PCB angioplasty were identified: (1) smaller acute gain at initial ballooning before PCB angioplasty; (2) geographic mismatch between PCB position and target recurrent PCI site during PCB angioplasty; and (3) PTCRA use at primary PCI.

Based on previous studies, ${ }^{15-18}$ DCB angioplasty is now a class I recommendation for the treatment of both BMSISR and DES-ISR in the European Society of Cardiology/ European Association for Cardio-Thoracic Surgery guidelines for repeat coronary revascularization. ${ }^{3}$ Nevertheless, recurrent ISR after DCB angioplasty still exists in the clinical setting. Several studies showing that the rate of recurrent ISR after PCB angioplasty is 4.3-21.1\%12,15-18 were consistent with the present data. The present LLL in the no recurrent ISR group was $0.28 \mathrm{~mm}$, which was also similar to prior studies (LLL, 0.03-0.46mm). ${ }^{7,12,15-18}$ Given that ISR after stent implantation can result in multiple metal layers, treatment options for recurrent ISR are limited. The present results may contribute to reduce recurrent ISR and achieve more effective and satisfactory outcome after PCB angioplasty.

We distinguished acute gain-1 and acute gain-2 to clarify the effect of initial BA. Acute gain-1 was significantly higher in the no recurrent ISR group compared with the recurrent ISR group, but there were no significant differences in acute gain- 2 between the 2 groups. Although post- 
procedure MLD was significantly different between the 2 groups $(2.51 \pm 0.45$ vs. $2.12 \pm 0.44, \mathrm{P}<0.001)$, we excluded this parameter from multivariate analysis because postprocedure acute gain-2 was not, which means that the difference in post-procedure MLD simply reflects the difference in the post-initial ballooning MLD. In the present study, lesion modification by the initial ballooning before PCB angioplasty played an important role in the PCB angioplasty because the PCB device itself is a simple intramural drug delivery device. Furthermore, on multivariate analysis, freedom from recurrent ISR for expansion rates $80-90 \%$ and $>90 \%$ was significantly higher than for the expansion rate $<80 \%$. This suggests that the threshold of expansion rate after initial $\mathrm{BA}$ is $\geq 80 \%$. In addition, using this threshold, freedom from recurrent ISR was significantly higher for expansion rate $\geq 80 \%$ than $<80 \%$. Expansion rate $\geq 80 \%$ after initial BA may prevent recurrent ISR, and optimal expansion may be responsible for the angiographic and clinical outcomes. In the present study, no significant differences were observed in initial balloon diameter or inflation pressure, or in the type of initial balloon catheter (non-compliant BA) between the recurrent ISR group and the no recurrent ISR group. Further prospective study is needed to achieve optimal expansion rate.

Geographic mismatch was significantly associated with recurrent ISR. The site of geographic mismatch treated by only initial ballooning is identical to the lesion treated by only conventional BA. Therefore, cases of geographic mismatch are predisposed to ISR, as supported by previous reports showing that conventional BA was significantly inferior to PCB angioplasty in clinical and angiographic outcomes. ${ }^{7,12,16,18}$

Initial calcium burden of the target lesion was also found to be related to recurrent ISR. Recurrent ISR occurred in half of the patients who required PTCRA at primary PCI due to severely calcified lesions. Moreover, PTCRA use at primary PCI was significantly higher in the recurrent ISR group than in the other group, and was an independent predictor of recurrent ISR. The target lesions in both groups (with or without PTCRA) had similar acute gain-1 after initial BA and there were no significant difference in acute gain-1 between the PTCRA group and the no PTCRA group, which suggests that the calcification itself may be responsible for the negative result after PCB angioplasty rather than insufficient expansion due to the calcification. ISR of originally severely calcified lesions requiring PTCRA may not be suitable for PCB angioplasty. During PCB angioplasty, almost all lesions in the PTCRA group were observed on IVUS or OCT, which showed no stent recoil, and that ISR was due to neointimal proliferation. Even if optimal expansion is obtained on initial ballooning in ISR with previous PTCRA use, we should consider the possibility of recurrent ISR after PCB angioplasty. The present results have raised a question as to whether DCB angioplasty should be avoided for an ISR lesion that has been treated with PTCRA at initial PCI. Further investigation is needed to clarify the possible advantage and disadvantage of DCB angioplasty and alternative strategies such as stent-in-stent technique or re-PTCRA.

In summary, 3 independent predictors of recurrent ISR after PCB angioplasty were identified: (1) smaller acute gain at initial ballooning before PCB angioplasty; (2) geographic mismatch; and (3) PTCRA use at primary PCI. The first 2 predictors can be overcome with careful bal- looning. The concept of "bigger is better" for lesion preparation before PCB angioplasty seems to be the key to prevent recurrent ISR. The last predictor pertains to the lesion characteristic itself, and history of PTCRA at primary PCI may be considered a risk factor of recurrent ISR. Careful attention to these factors should improve the success rate after PCB angioplasty.

\section{Study Limitations}

The present study has several limitations. First, this was a small observational analysis from a single center with a retrospective 2-arm design. Further randomized studies enrolling a larger number of cases are needed to clarify this problem. Second, this study protocol included both BMSISR and DES-ISR despite the differences in the efficacy of PCB angioplasty according to type of restenotic stent. ${ }^{11,12}$ Third, comparison of tissue morphology on intravascular imaging is required, given the reported association between tissue morphology and clinical outcomes. ${ }^{19}$ Fourth, although mechanisms of ISR such as under-expansion and stent fracture have been reported, ${ }^{2}$ we did not consider these problems and their influence on subsequent ISR. Finally, the factors of medication before and after the procedure, which may affect re-atherosclerosis, should also be addressed.

\section{Conclusions}

Recurrent ISR occurred in $13.9 \%$ of lesions after PCB angioplasty for ISR. And (1) optimal expansion at initial ballooning before PCB angioplasty $\geq 80 \%$; (2) careful positioning of the PCB catheter to avoid geographic mismatch; and (3) lower calcium burden at primary PCI are important clinical factors to prevent recurrent restenosis after PCB angioplasty for ISR.

\section{Disclosures}

The authors declare no conflict of interest.

\section{References}

1. Sarno G, Laqerqvist B, Frobert O, Nilsson J, Olivecrona G, Omerovic E, et al. Lower risk of stent thrombosis and restenosis with unrestricted use of 'new-generation' drug-eluting stents: A report from the nationwide Swedish Coronary Angiography and Angioplasty Registry (SCAAR). Eur Heart J 2012; 33: 606-613.

2. Dangas GD, Claessen BE, Caixeta A, Sanidas EA, Mintz GS, Mehran R. In-stent restenosis in the drug-eluting stent era. $J$ Am Coll Cardiol 2010; 56: 1897-1907.

3. Windecker S, Kolh P, Alfonsoet F, Collet JP, Cremer J, Falk V, et al. 2014 ESC/EACTS Guideline on myocardial revascularization. Eur Heart J 2014; 35: 2541-2619.

4. Mehilli J, Byrne RA, Tiroch K, Pinieck S, Schulz S, Kufner S, et al. Randomized trial of paclitaxel- vs. sirolimus-eluting stents for treatment of coronary restenosis in sirolimus-eluting stents: The ISAR-DESIRE 2 (Intracoronary Stenting and Angiographic Results: Drug Eluting Stents for In-Stent Restenosis 2) study. $J$ Am Coll Cardiol 2010; 55: 2710-2716.

5. Alfonso F, Garcia J, Perez-Vizcayno MJ, Hernando L, Hernandez $\mathrm{R}$, Escaned J, et al. New stent implantation for restenosis after stenting for in-stent restenosis: Implications of a third metal layer in human coronary arteries. J Am Coll Cardiol 2009; 54: $1036-1038$

6. Komatsu R, Ueda M, Naruko T, Kojima A, Becker AE. Neointimal tissue response at sites of coronary stenting in humans. Circulation 1998; 98: 224-233.

7. Byrne RA, Neumann FJ, Mehilli J, Pinieck S, Wolff B, Tiroch $\mathrm{K}$, et al. Paclitaxel-eluting balloons, paclitaxel-eluting stents, and balloon angioplasty in patients with restenosis after implantation of a drug-eluting stent (ISAR-DESIRE 3): A randomised, openlabel trial. Lancet 2013; 381: 461-467. 
8. Indermuehle A, Bahl R, Lansky AJ, Froehlich GM, Knapp G, Timmis A, et al. Drug-eluting balloon angioplasty for in-stent restenosis: A systematic review and meta-analysis of randomised controlled trials. Heart 2013; 99: 327-333.

9. Lee JM, Park J, Kang J, Jeon KH, Jung JH, Lee SE, et al. Comparison among drug-eluting balloon, drug-eluting stent, and plain balloon angioplasty for the treatment of in-stent restenosis: A network meta-analysis of 11 randomized, controlled trials. JACC Cardiovasc Interv 2015; 8: 382-394.

10. Siontis GC, Stefanini GG, Mavridis D, Siontis KC, Alfonso F, Pérez-Vizcayno MJ, et al. Percutaneous coronary interventional strategies for treatment of in-stent restenosis: A network metaanalysis. Lancet 2015; 386: 655-664.

11. Wohrle J, Zadura M, Mobius-Winkler S, Leschke M, Opitz C, Ahmed W, et al. SeQuent Please World Wide Registry: Clinical results of SeQuent Please paclitaxel-coated balloon angioplasty in a large-scale, prospective registry study. $J$ Am Coll Cardiol 2012; 60: $1733-1738$

12. Habara S, Iwabuchi M, Inoue N, Nakamura S, Asano R, Nanto $\mathrm{S}$, et al. A multicenter randomized comparison of paclitaxelcoated balloon catheter with conventional balloon angioplasty in patients with bare-metal stent restenosis and drug-eluting stent restenosis. Am Heart J 2013; 166: 527-533.

13. Scheller B, Clever YP, Kelsch B, Hehrlein C, Bocksch W, Rutsch $\mathrm{W}$, et al. Long-term follow-up after treatment of coronary instent restenosis with a paclitaxel-coated ballo on catheter. JACC Cardiovasc Interv 2012; 5: 323-330.

14. Kufner S, Cassese S, Valeskiniet M, Neumann FJ, Schulz-Schüpke $\mathrm{S}$, Hoppmann P, et al. Long-term efficacy and safety of paclitaxel-eluting balloon for the treatment of drug-eluting stent restenosis: 3-year results of a randomized controlled trial. JACC Cardiovasc Interv 2015; 8: 877-884.

15. Habara S, Kadota K, Shimada T, Ohya M, Amano H, Izawa Y, et al. Late restenosis after paclitaxel-coated balloon angioplasty occurs in patients with drug-eluting stent restenosis. $\mathrm{J}$ Am Coll Cardiol 2015; 66: 14-22.

16. Rittger H, Brachmann J, Sinha AM, Waliszewski M, Ohlow M, Brugger A, et al. A randomized, multicenter, single-blinded trial comparing paclitaxel-coated balloon angioplasty with plain balloon angioplasty in drug-eluting stent restenosis: The PEPCADDES study. J Am Coll Cardiol 2012; 59: 1377-1382.

17. Unverdorben M, Vallbracht C, Cremers B, Heuer H, Hengstenberg C, Maikowski C, et al. Paclitaxel-coated balloon catheter vs. paclitaxel-coated stent for the treatment of coronary in-stent restenosis. Circulation 2009; 119: 2986-2994

18. Habara S, Mitsudo K, Kadota K, Goto T, Fujii S, Yamamoto $\mathrm{H}$, et al. Effectiveness of paclitaxel-eluting balloon catheter in patients with sirolimus-eluting stent restenosis. JACC Cardiovasc Interv 2011; 4: 149-154.

19. Tada T, Kadota K, Hosogi S, Miyake K, Ohya M, Amano H, et al. Association between tissue characteristics assessed with optical coherence tomography and mid-term results after percutaneous coronary intervention for in-stent restenosis lesions: A comparison between balloon angioplasty, paclitaxel-coated balloon dilation, and drug-eluting stent implantation. Eur Heart $J$ Cardiovasc Imaging 2015; 10: 1001-1011.

\section{Supplementary Files}

\section{Supplementary File 1}

Table S1. QCA of calcified lesions vs. presence of PTCRA at primary PCI

Please find supplementary file(s);

http://dx.doi.org/10.1253/circj.CJ-17-0095 